\title{
Protein Serine/Threonine Phosphatase
}

National Cancer Institute

\section{Source}

National Cancer Institute. Protein Serine/Threonine Phosphatase. NCI Thesaurus. Code C34078.

Encoded by Protein Serine/T hreonine Phosphatase Genes, Protein Serine/T hreonine Phosphatases are a group of enzymes that specifically dephosphorylate phosphoserine and phosphothreonine residues in specific target proteins. Together with serine/threonine protein kinases, these enzymes regulate serine and threonine phosphorylation and dephosphorylation of target proteins in cellular signal transduction pathways typically involved in cell growth control. 